\title{
Estudio comparativo de guiones culturales brasileños y españoles desde la perspectiva de la etnopragmática
}

\author{
Marta Giralt Lorenz ${ }^{1}$ (GIRALT LORENZ, Marta) \\ Master y DEA en Didáctica de lenguas y literatura \\ Instituto Cervantes de Brasilia \& Universidad de Barcelona \\ giraltmarta@gmail.com
}

\section{Resumen}

Este artículo tiene como objetivo presentar los resultados obtenidos tras la realización de un estudio comparativo entre guiones culturales brasileños y españoles. A partir del modelo teórico de guiones culturales que nos ofrece la etnopragmática, este estudio analiza y compara guiones de despedida y negación en hablantes de portugués y en hablantes de español. Asimismo, se analiza una situación concreta de interacción social para determinar si existen diferencias entre ambas formas de comunicación e interacción cultural. Finalmente, las conclusiones del estudio demuestran las diferencias en los guiones culturales de cada una de las culturas analizadas y las formas de interacción de ambas.

Palabras clave: lengua, cultura, guiones culturales, comunicación intercultural

\begin{abstract}
The aim of this article is to present the results of a study that compares Brazilian and Spanish cultural scripts. Based on the cultural scripts theoretical framework offered by the ethonopragmatics, our study analyses and compares cultural scripts (saying goodbye and negating) in Portuguese and Spanish speakers. At the same time, we also analyze a situation of social interaction between speakers of both cultures to see any cultural difference between both communication and interaction patterns.

Finally, we present the conclusions that show the differences in the cultural script of each culture and their social interaction forms.
\end{abstract}

Keywords: language, culture, cultural scripts, intercultural communication

1 Profesora de plantilla del Instituto Cervantes de Brasilia y miembro colaborador del grupo de investigación del departamento de Didáctica de la Lengua y la Literatura de la Universidad de Barcelona dirigido por el Dr. Francisco Cantero Serena. 


\section{Introducción}

La motivación del estudio, en la que se basa este artículo, surgió como consecuencia de las semejanzas que existen entre la cultura española y la cultura brasileña en relación a las formas de interacción social y de comportamiento de los hablantes de ambas culturas.

A pesar de estas similitudes que, a simple vista, pueden parecer una realidad, existen formas de comportamiento, de interacción y manifestaciones lingüísticas que difieren mucho entre las dos culturas. Obviamente las lenguas de comunicación que se utilizan en cada una de estas culturas son diferentes, pero creemos que además de esa diferencia existen también grandes divergencias en cuanto a los guiones culturales que poseen cada una de ellas para las mismas situaciones de interacción social.

Los estudios de comunicación intercultural suelen otorgar más atención a culturas que presentan grandes diferencias entre sí y en las que, por tanto, sus formas de interacción y de comunicación pueden verse amenazadas por los malosentendidos culturales, o simplemente, por la falta de comunicación.

Los conocimientos que se pueden tener de una cultura ayudan a entender lo que sucede alrededor, ayudan a actuar adecuadamente y a desenvolverse en un contexto cultural concreto y dan la competencia necesaria para interactuar con los miembros de una determinada comunidad cultural.

Esta tercera dimensión relacionada con la interacción social, ha sido precisamente la que más nos ha interesado en este estudio puesto que creemos que los individuos adscritos a una cultura determinada y a una lengua, comparten y dan por sobreentendido una serie de valores, creencias, símbolos, maneras de ver el mundo, presuposiciones y actuaciones ante las que un hablante, que no pertenezca a esa cultura y que no comparta esa lengua, puede sentirse ajeno.

Desde esta perspectiva, la cultura brasileña y la cultura española se sitúan en una zona de proximidad, tanto cultural como ligüística, que muchas veces puede parecer mucho menor de lo que realmente es. Precisamente, este ha sido el motivo principal que nos instigó a realizar este breve estudio comparativo en el que se pondrán de relieve posibles diferencias o similitudes entre ambas culturas en el ámbito de las interacciones sociales, partiendo del modelo teórico que ofrece la etnopragmática. 


\section{Marco teórico: los guiones culturales y el paradigma de la etnopragmática}

En el momento de escoger el marco teórico en el que se basa este estudio, tuvimos en cuenta cuál podría ser el paradigma que mejor se ajustaría al análisis de la comunicación intercultural, y que al mismo tiempo también considerara la interacción social entre los distintos hablantes de una comunidad lingüístico-cultural. La cuestión era encontrar un modelo de análisis que contemplara acciones, estilos y rituales de interacción.

El paradigma de la etnopragmática se nos presenta como un tipo de abordaje de las manifestaciones culturales donde es importante el sentido de los intercambios comunicativos, al mismo tiempo que se incluyen cuestiones como: los valores, las creencias, las actitudes, las categorías sociales y las emociones. La etnopragmática, también denominada pragmática cross-cultural, considera que los hablantes de culturas diferentes hablan y realizan intercambios comunicativos de forma diferente porque piensan, sienten y se relacionan con los otros miembros de manera diferente. Por tanto, se da por sentado que las sociedades hablan de una forma u otra e interactúan de una manera determinada de acuerdo con ciertos valores culturales y unas prioridades culturales que varían de una cultura a otra (GOODARD; WIERZBICKA, 2004).

Tomando estos principios como marco general, la etnopragmática se aleja de consideraciones universalistas (principios universales de comunicación; modelos universales de faces o inventarios universales de tipos de actos de habla), y sienta sus fundamentos en la existencia de herramientas universales de análisis. Cada cultura posee sus conceptos clave, los cuales pueden formularse mediante un lenguaje sencillo en forma de guiones culturales. De esta manera, las explicaciones de dichos conceptos y la forma de cómo éstos quedan representados en interacciones e intercambios lingüístico-culturales, son abordados, percibidos e interpretados desde la perspectiva del miembro que pertenece a la cultura y lengua analizadas, pero al mismo tiempo, son entendidos por los miembros ajenos a esa cultura. Por tanto, el acercamiento que nos proporciona la etnopragmática queda libre de cualquier acusación de etnocentrismo, y por otro lado se nos presenta como un modo muy acertado de explicar las especificidades de fenómenos pragmático-culturales.

Las herramientas de análisis en las que se asienta la etnopragmática son otro de los puntos clave que circunvalan el acercamiento al análisis de la comunicación intercultural, motivo por el cual es necesario determinarlas conceptualmente. 


\section{El metalenguaje semántico natural}

Denominada Natural Semántica Metalanguage Theory, esta teoría está basada en un programa de investigación que cuenta ya con más de tres décadas de existencia. Grosso modo, la teoría defiende que todas las lenguas comparten un repertorio de significados, partículas conceptuales, denominadas Semantic primes $^{2}$, y que dichos significados tienen unos exponentes lingüísticos determinados, compartiendo todos ellos una gramática de combinación y complementación. De este modo, todas las lenguas comparten un vocabulario central común que sería suficiente para referirse a conceptos y palabras más complejos que existieran en cualquier lengua y cultura. Los primitivos semánticos que establecen Goddard y Wierzbicka (2002) están constituidos por 60 ítems y sus respectivos dominios (véase anexo I).

Los primitivos semánticos permiten una combinación para formar frases, que son las afirmaciones que permiten la articulación inteligible de los cultural scripts ${ }^{3}$, los cuales son el siguiente paso a seguir para llegar al entendimiento de las practicas discursivas desde una perspectiva cultural libre de cualquier etnocentrismo.

Llegados a este punto, nos gustaría señalar tres de los motivos ${ }^{4}$ por los que creemos que seguir los presupuestos del Metalenguaje Semántico Natural y hacer uso de primitivos semánticos, es adecuado y óptimo para nuestro estudio:

1. Desde la perspectiva del Metalenguaje Semántico Natural, conceptos complejos pueden ser explicados a partir de grupos simples de elementos.

2. El grupo de primitivos semánticos posee equivalencia en todas las lenguas, de ahí que puedan ser considerados universales lingüísticos.

3. Los primitivos semánticos poseen neutralidad cultural.

Tras la descripción de esta primera herramienta metodológica que ofrece la etnopragmática, pasaremos a continuación a hablar de los guiones culturales como categoría de análisis.

\footnotetext{
${ }^{2}$ En este estudio hemos optado por referirnos a los semantic primes como primitivos semánticos.

${ }^{3}$ En este estudio hemos optado por referirnos a los cultural scripts como guiones culturales.

${ }^{4}$ Las razones mencionadas coinciden con algunas de las razones a las que alude Escalante Alvarez (2009) al justificar el modelo de análisis escogido para su investigación.
} 


\section{Guiones culturales}

Los guiones culturales emergen como una técnica para la descripción de actitudes, valores, normas y presuposiciones pertenecientes a una cultura. Todos esos rasgos y características de una determinada cultura se perciben como conocimiento compartido entre las personas de esa cultura y comunidad de hablantes. Es importante hacer hincapié en el hecho de que estas afirmaciones se conciben como conocimiento compartido desde una perspectiva neutra y simple. Los elementos que componen los guiones culturales surgen intuitivamente en forma de paráfrasis auto-explicativa, conservando a su vez un cierto rigor empírico.

Sin embargo, no debemos olvidar que las sociedades son heterogéneas y no todos sus miembros tienen que suscribirse a los guiones y las reglas culturales, pero es necesario que estén familiarizados con éstos y formen parte de su conocimiento para la interpretación de las prácticas discursivas en un contexto cultural determinado. Asimismo, no podemos dejar de lado las diferencias regionales y las variaciones sociales en una misma cultura y comunidad lingüística. Los guiones culturales, van de lo más general a la descripción minuciosa de la forma de interactuar, reflejando creencias culturales. También pueden referirse a comunicación no verbal, gestos, lenguaje corporal, proxémica.

Pese a ello, los guiones culturales pueden ser considerados como una categoría de análisis para describir y formular reglas culturales al mismo tiempo que, como afirman Goddard y Wierzbicka (1994), poseen la capacidad de esclarecer las diferencias culturales, incluidas aquellas que afectan más directamente a los estilos de comunicación.

Después de esta aproximación a las herramientas y categorías de análisis en las que se va a basar el estudio que presentamos en este artículo, pasaremos al siguiente apartado en el que trataremos de manera muy sucinta sobre los objetivos y la metodología de trabajo.

\section{Objetivos y metodología de trabajo}

Los objetivos de esta investigación van a ceñirse a responder algunas preguntas que nos hemos planteado al comparar tres situaciones de la cultura brasileña y de la cultura española. 
Las cuestiones en concreto podrían enunciarse de la siguiente manera:

1. ¿Existen diferencias concretas en la forma en la que se despiden los españoles y la forma en la que lo hacen los brasileños? ¿Qué guión cultural se esconde detrás?

2. ¿Qué guión cultural y qué valores, creencias y presupuestos sociales existen en una situación concreta de interacción social? En este caso la situación concreta escogida sería la forma de pagar una cuenta común en un restaurante después de una cena con un grupo de amigos. ¿Comparten la cultura brasileña y la cultura española esos guiones, valores y creencias?

3. ¿De qué forma un hablante lleva a cabo el rechazo a una petición realizada por su interlocutor? ¿Comparten la cultura brasileña y la cultura española el mismo guión cultural en esta situación de interacción? ¿Los presupuestos, las formas de actuación y las manifestaciones lingüísticas que se dan en esta situación son las mismas para ambas culturas?

Para responder estas preguntas, primeramente, vamos a plantear 3 situaciones comunicativas y 2 posibles guiones culturales para cada una de ellas. Dichas situaciones se han creado y diseñado como un modelo a priori del que vamos a partir y que vamos a elicitar a partir de los cuestionarios.

Para comprobar si funcionan los guiones culturales creados para describir cada una de las situaciones específicas de comunicación y de interacción entre dos interlocutores, vamos a pasar por escrito la descripción de cada uno de los guiones ${ }^{5}$ a 6 personas españolas y a 6 personas brasileñas.

Se trata de que cada persona marque con qué guión cultural se identifica más y que justifique su elección. Además, añadiremos un pequeño apartado en el que se pueda hacer algún matiz en relación a distintos contextos que pudieran hacer variar la elección de la respuesta que se ha escogido, así como posibles sugerencias sobre qué modificaciones realizarían ellos respecto al guión cultural con el que están identificándose.

A partir de los resultados obtenidos podremos llegar a algunas conclusiones de carácter muy general sobre las semejanzas y las similitudes de las distintas formas de interactuar entre hablantes y miembros de la cultura española y la cultura brasileña.

\footnotetext{
${ }^{5}$ Ver modelo de ficha en Anexo 2.
} 
De igual modo, vamos a poder detectar si hay alguna diferencia relevante en las manifestaciones lingüísticas que se llevan a cabo en cada una de las tres situaciones y los guiones culturales de éstas.

Finalmente, será posible determinar cuál puede ser el guión cultural con el que mayor número de personas de cada una de las culturas se ha identificado más, pudiendo establecerse una posible legitimación de éste.

\section{Guiones culturales usados en este estudio y resultados obtenidos}

Los tres guiones culturales que hemos diseñado para este estudio van a referirse a tres situaciones y actos de habla concretos:

a. Las despedidas.

b. La forma de pagar una cuenta común en un bar entre un grupo de personas.

c. Denegar una petición.

Para cada una de ellas vamos a describir el guión cultural intentando seguir la metodología que recomienda la etnopragmática. Tras dicho guión, concretizaremos la situación de cada uno y detallaremos paso a paso como se desarrolla la interacción en cada caso.

Después de la presentación de cada situación, vamos a comentar los resultados obtenidos en los 12 cuestionarios que completaron nuestros doce informantes, 6 de los cuales eran españoles y 6 brasileños. Para ir comentando los datos, tomaremos cada una de las situaciones por separado para ver qué elección fue la más mayoritaria en cada uno de los grupos y qué tipo de comentarios añadieron los informantes.

\section{Situación 1: Las despedidas.}

En muchas culturas la separación se percibe como algo doloroso, por tanto, cuando una persona se despide de otra tiene que seguir todo un ritual previo a la separación. El momento de la separación debe prolongarse al máximo. Creemos que los hablantes y miembros de un tipo de cultura así, que no siguen el guión cultural que presentamos a continuación y que tiene formas de actuación distintas a las descritas en la situación de despedidas, son miembros de la comunidad que van a poseer una imagen social anormal, pero sin que llegue a ser negativa. Es decir, preservan su face positiva, 
aunque pueden llegar a ser vistos como un poco antisociales ya que están quebrantando alguna de las normas establecidas en una situación específica. De todas formas, el incumplimiento de esta norma social, no es una agresión social de alto grado.

Los guiones culturales propuestos son:

1. "Las personas piensan que la separación es dolorosa. El momento de la separación debe prolongarse al máximo y debe atenuarse programando un nuevo encuentro".

2. "Las personas no piensan que la separación sea dolorosa. El momento de la separación no tiene que seguir ningún ritual y puede ser breve".

Situación de despedida:

1-Dos amigos acaban de estar tomando un café. Hacía algún tiempo que no se veían. Uno de ellos debe marcharse y es el que inicia la despedida. La persona que tiene que marcharse debe introducir de forma indirecta un motivo que va a servir como justificación para iniciar el ritual previo a la separación y despedida. Muchas veces puede ser una señal tan sutil como mirar el reloj.

$\mathrm{Si}$ su interlocutor no reconoce ese intento, se debe insinuar de nuevo, siendo cada vez más concreto. En el momento en que el interlocutor reconoce que se van a despedir, se inicia el preludio a la separación: proponer un futuro encuentro, pero sin cerrarlo; respetar siempre que si uno de los participantes inicia un nuevo tema, su interlocutor debe continuarlo; enviar saludos a personas conocidas por ambos...

2-Dos amigos acaban de estar tomando un café. Hacía algún tiempo que no se veían. Uno de ellos debe marcharse y es el que inicia la despedida. Se lo comunica a su interlocutor y ambos inician la despedida promoviendo un posible encuentro para una próxima vez pero sin extender demasiado el intercambio.

\section{Resultados obtenidos en la situación 1:}

En esta primera situación los resultados entre las respuestas de los informantes brasileños y los españoles han sido inversamente proporcionales. Mientras que 4 de los 6 informantes brasileños se han identificado con la descripción 2 de la primera situación, 4 de 6 españoles lo han hecho con la descripción 1.

Huelga decir que los dos informantes españoles que han optado por la opción 2, han añadido unos matices en sus comentarios que dejan entrever como en realidad también se identifican con la situación 1. De hecho el informante 6 alude a la forma en que llevaría a cabo la interacción en la situación 2 para no ofender a su interlocutor, lo que presupone que esa actuación podría ofenderlo.

Por otro lado, en el grupo de informantes brasileños, uno de los que opta por la descripción 1 alude a argumentos relacionados con la "elegancia" y "educación" que 
reflejan esa actuación concreta en la interacción social. Por tanto, dicha informante parece poseer su propia percepción de la imagen social.

De la misma manera, entre los informantes españoles se habla también de actuación "mal educada" y de "quedar mal", y por tanto, se está haciendo referencia a la necesidad de actuar siguiendo la norma social implícita en la interacción de la situación 1 si se quiere preservar la face positiva del hablante y miembro de la sociedad.

En cuanto a las posibles variaciones de actuación en dicha situación 1, se señala que el grado de amistad y de confianza que exista entre los interlocutores va a poder determinar el tipo de actuación que se lleve cabo en una situación de despedida entre dos amigos.

Otra cuestión que ha surgido del análisis de los cuestionarios en torno a esta primera situación, es la alusión de alguno de los informantes a su preferencia por actuar de forma directa y sin rodeos. Resulta un tanto contradictorio que en esta situación, el valor de la sinceridad y de decir las cosas de forma directa, sea percibido como algo positivo, cuando en muchas otras situaciones, por ejemplo en la situación 3 de este estudio, si la sinceridad o honestidad puede ofender se utilizan mecanismos para evitarla.

Tal vez, podríamos aventurarnos a decir que el valor del dolor en la separación y en la despedida no se comparte entre la cultura brasileña y la cultura española.

Así pues, esta primera situación presenta unos resultados muy coherentes y significativos, de forma que nos ha sido posible poder observar todos los fenómenos y cuestiones que acabamos de comentar.

\section{Situación 2: la forma de pagar una cuenta común en un bar entre un grupo de personas.}

En muchas culturas existe la creencia social de que es bueno ser generoso y que es importante preservar esa imagen social Por tanto, cualquier situación en la que peligre la face positiva del individuo miembro de esa cultura, en relación al valor de la generosidad, va a estar muy pautada y va a tener un guión cultural muy específico.

Los guiones culturales propuestos son:

1. "Es importante lo que las personas piensan de mi. Las personas creen que es bueno ser generoso. No es bueno que las personan piensen que no soy generoso". 
2. "Es importante lo que las personas piensan de mi. Las personas no creen que el valor de la generosidad sea importante. No es importante si las personan piensan que no soy generoso".

Situación de pagar una cuenta común en un bar entre un grupo de personas: 1-Un grupo de amigos acaba de cenar en un restaurante y llega el momento de pagar la cuenta. La persona que agarra la nota divide el total de la cuenta entre el número de personas que hay en la mesa, sin tener en cuenta qué ha consumido cada uno de ellos.

En alguna situación específica, si el grupo de amigos tiene una relación muy intima, pueden producirse rituales de lucha por asumir el pago de la cuenta.

En una situación de este tipo, el hecho de mostrar preocupación por quién paga más o menos, puede ser considerado una evidencia de que no se es generoso, característica que socialmente está sancionada y que daña seriamente la imagen social de la persona.

La forma de pagar en un restaurante es una norma social que es preciso respetar si no se quiere perder prestigio y respeto social.

2-Un grupo de amigos acaba de cenar en un restaurante y llega el momento de pagar la cuenta. Cada persona paga su parte de la cuenta de acuerdo con lo que ha consumido.

En esta situación, el que una persona muestre preocupación por pagar solamente lo que ha consumido, evitando así pagar más de lo que debe, no es considerada un comportamiento socialmente inadecuado y sancionado. No daña la face del individuo, ya que, el valor de la generosidad en un contexto como éste, o de la despreocupación por quién paga más o menos, no tiene gran relevancia en esta cultura.

Resultados obtenidos en la situación 2.

Esta segunda situación es la que presenta menos diferencias en las respuestas que han dado los informantes. Todos los informantes españoles se han identificado con la descripción 1 de esta segunda situación, mientras que todos los informantes brasileños, excepto una ${ }^{6}$, lo han hecho con la descripción 2.

Ante estos resultados podría afirmarse que nos encontramos ante una forma de interacción social muy distinta entre ambas culturas.

Los comentarios que aportan los informantes españoles (comportamiento "un poco feo", "mal educado" "estar mal visto") confirman que existe una norma social implícita en dicha situación, norma que se sustenta en el valor de la generosidad. Como ejemplo de ello, encontramos un comentario que hace referencia a cómo el grupo es el

\footnotetext{
${ }^{6}$ A pesar de que esta informante es la única que ha optado por la descripción 1, sus comentarios al respecto coinciden con los que han realizado algunos de los informantes españoles (en concreto cuando hablan de que una excepción a pagar la cuenta a partes iguales es cuando la diferencia entre lo que han consumido unos y otros es especialmente grande). Por tanto, puede que en el caso de esta informante, sus valores y creencias se compartan con las de los españoles.
} 
que acepta que se haga una diferenciación en la forma de pago, y nunca es la persona a la que le toca pagar menos.

En cuanto a los informantes brasileños, hemos encontrado un comentario (literalmente el comentario ha sido "es lo más justo”) que se distancia totalmente del valor de la generosidad como actitud de prestigio social. Es significativo también que dos de las informantes brasileñas comenten que esta forma de actuar en el pago de una cuenta es una cuestión cultural típica de Brasil. Con esta afirmación están dando por hecho que en muchas otras culturas pueda ser diferente.

En cuanto a los distintos contextos que harían variar la elección de la descripción de esta segunda situación que ha realizado cada informante, tanto los brasileños como los españoles aluden a contextos formales de negocios y al grado de amistad y de cercanía que exista entre las personas que se encuentran en la situación. A su vez, se hace hincapié en distintas circunstancias en las que podría variar esa forma de pagar: un amigo invita para celebrar alguna cosa, se paga por turnos cada vez que los amigos se ven... Todas estas variaciones quedarían un poco al margen de la situación que se ha descrito en nuestro guión cultural. Obviamente, pueden existir infinidad de circunstancias que presenten una forma de actuar u otra.

\section{Situación 3: denegar una petición.}

En muchas culturas existe la creencia de que denegar una petición es una agresión al interlocutor, y que es necesario atenuar la denegación. En algunos casos esos atenuantes van a venir dados por una fuerte argumentación y justificación (de manifestación lingüística) que explique los motivos por los que se ha producido la negativa a la petición. Si se consigue ese cometido, la denegación es aceptada por el interlocutor y éste no se siente agredido, aceptándola.

Por el contrario, en muchas otras culturas, el rechazo de una petición es una ofensa, y por tanto, la negación a una petición no es posible. Es necesario el uso de atenuadores (de manifestación lingüística) y de estrategias para poder hacer entender al interlocutor que la petición no va a ser aceptada, pero sin realizar la negación de forma directa.

Los guiones culturales van a ser:

1. "Las personas se sienten mal cuando reciben una negativa sin explicación. Si digo no a una persona debo explicar muy bien por qué". 
2. "Las personas se sienten muy mal cuando reciben una negativa. No puedo negar de forma directa para no ofender a las personas".

\begin{abstract}
Situación en al que se deniega una petición:
1-Un compañero de trabajo le pide a otro compañero, al cual solo conoce del contexto laboral, si le puede ayudar a terminar un informe que debe presentar al día siguiente. Ante esta petición, su compañero se justifica diciéndole que no puede ayudarle porque tiene que hacer otra cosa. En su argumentación, se asegura de justificar debidamente y con motivos más que suficientes su negación. Se disculpa repetidas veces, al mismo tiempo que se asegura que su interlocutor ha aceptado la negación y que no la ha sentido como una agresión u ofensa.

2- Un compañero de trabajo le pide a otro compañero, al cual solo conoce del contexto laboral, si le puede ayudar a terminar un informe que debe presentar al día siguiente. Ante esta petición, su compañero le plantea otras opciones que le pueden ser útiles para terminar su informe. Si su interlocutor insiste, se va a ver obligado a decir siempre de forma indirecta que no va a poder, evitando en todo momento realizar una negación clara y evidente.
\end{abstract}

Resultados obtenidos en la situación 3.

De las tres situaciones analizadas, esta tercera es la que presenta más disparidad en las respuestas que han dado los informantes brasileños. En cambio, los informantes españoles han coincidido todos en identificarse con la descripción 1.

Un hecho importante es que los informantes brasileños que han escogido la situación igual a la de los españoles, han realizado unos comentarios muy distintos a los comentarios de los españoles.

En el caso de los informantes españoles, parece que se valora la honestidad y el ser directo, al mismo tiempo que se ve como algo necesario que exista una justificación bien argumentada que sustente la negación de la petición y que permita que en la interacción esté todo más claro. Por este motivo, en este tipo de interacción aparecen unas evidencias lingüísticas muy específicas, como por ejemplo en el caso de la justificación, el uso de exponentes lingüísticos como es que.

Por el contrario, los informantes brasileños hacen referencia al valor ofensivo que tiene la negación y que, por tanto, obliga a que la justificación esté muy bien construida.

Por tanto, informantes de ambas nacionalidades usan la justificación, aunque en el caso de los brasileños parece que con un fin mucho más concreto.

Junto a todo esto, encontramos también a tres informantes brasileños que exponen de manera explícita como la negación es una ofensa de alto grado y hay que 
evitarla pese a que eso signifique no ser directo. Por ese motivo, estos tres informantes se identifican con la descripción 2 de la situación 3.

Hasta aquí llegarían las tres situaciones analizadas. Cada una de ellas nos muestran distintas formas de enfrentarse a una misma situación de interacción social, con todos los matices, idiosincrasias de los hablantes, sus presuposiciones, sus valores y sus creencias. A partir de su análisis y de la reflexión de los resultados extraídos de los datos empíricos provenientes de las cuestionarios es posible concluir este artículo con algunas consideraciones que presentamos en el último apartado.

\section{Conclusiones}

Como conclusiones de este estudio, en primer lugar, nos gustaría mencionar que los guiones culturales que se elaboraron para describir las tres situaciones de interacción han funcionado y nos han permitido poder contestar alguna de las preguntas que nos habíamos planteado.

Podemos considerar que la cultura española y la cultura brasileña difieren en algunas formas de interacción, entre ellas las despedidas y la forma de pagar la cuenta en un restaurante cuando un grupo de amigos va a cenar. Los guiones culturales que poseen cada una de estas interacciones son distintos, así como los valores y las creencias que se esconden detrás de éstos.

Al elaborar los guiones culturales realizamos una serie de reflexiones acerca de valores tales como la generosidad o la ofensa ante la negación. Tras finalizar el estudio, han emergido otros valores, como por ejemplo el de la honestidad, al que no se había hecho referencia, pero que ha aparecido al analizar los datos.

Así pues, podemos afirmar que entre las interacciones brasileñas y las españolas existen diferencias significativas, y por tanto, ambas culturas poseen unos guiones culturales distintos. El desconocimiento de éstos puede llevar muchas veces a malosentendidos culturales graves, pues detrás de estos guiones se esconden valores, creencias y normas sociales de fuerte arraigo cultural.

Nos gustaría que este estudio motivara futuras investigaciones y estudios en los

que se analice de forma más amplia esa variedad cultural de la que gozan la cultura 
española y brasileña, dos culturas que pese a tener muchas cosas en común, poseen al mismo tiempo una gran distancia socio-cultural.

Será precisamente mediante la comunicación intercultural que las diferencias se presentarán como enriquecedoras y no como una fuente de malosentendidos culturales. Por eso, vale la pena que sigamos la senda que nos va a permitir trazar esos puentes entre culturas.

\section{Bibliografía}

ESCALANTE ALVAREZ, Alba E.. Roteiros culturais, frames e metáforas conceituais: abordagens para o estudo da unidade/diversidade linguística/cultural dos falantes da língua española. 2009. Dissertação (Mestrado em Lingüística Aplicada) - Instituto de Letras, Universidade de Brasilia, Brasília, 2009. Disponível em:

$<$ http://www.pgla.unb.br/index.php?option=com_content\&view=category\&layout=blog \&id=10\&Itemid=54>.

GODDARD, Cliff; WIERZBICKA, Anna. Semantic and lexical universals: theory and empirical findings. Amsterdam/Filadelfia: John Benjamins, 1994.

GODDARD, Cliff; WIERZBICKA, Anna. Discourse and culture. In: DIJK Teun v. (Ed.) Discourse as a social interaction. London: Sage, 1997.

GODDARD, Cliff; WIERZBICKA, Anna. (Eds.) Meaning and universal grammar: theory and empirical findings. Amsterdam/Philadelphia: John Benjamins, 2002.

GODDARD, Cliff; WIERZBICKA, Anna. Cultural scripts: what are they and what are they good for? Intercultural Pragmatics, v. 1, n. 2, p. 153-166, 2004.

\section{Anexo I}

\begin{tabular}{|ll|}
\hline Substantives & I, you, someone/person, people \\
Relational substantives & something, thing, body, part \\
Determiners & this, the same, other \\
Quantifiers & one, two, some, all, many/much \\
Evaluators & good, bad \\
Descriptors & big, small \\
Mental/experiential predicates & think, know, want, feel, see, hear \\
\hline
\end{tabular}




\begin{tabular}{|ll|}
\hline Speech & say, words, true \\
Actions and events & do, happen, move \\
Existence and possession & there is/exist, have \\
Life and death & live, die \\
Time & $\begin{array}{l}\text { when/time, now, before, after, a long } \\
\text { time, a short time, for some time, } \\
\text { moment. }\end{array}$ \\
Space & $\begin{array}{l}\text { where/place, here, above, below, far, } \\
\text { near, side, inside, touch (contact) }\end{array}$ \\
Logical concept & not, maybe, can, because, if \\
Intensifier, aumentor & very, more \\
Similarity & like/way \\
\hline
\end{tabular}

(GODDARD; WIERZBICKA, 2002)

\section{Anexo II}

Este segundo anexo es la ficha modelo que se ha utilizado para conseguir los datos que informantes brasileños e informantes españoles nos suministraron.

A cada uno de los informantes se le entregó la ficha correspondiente a cada situación, es decir, tres situaciones.

\section{Edad: \\ Nacionalidad: \\ Profesión:}

Marca con una cruz con que descripción de las tres situaciones te identificas más y responde las cuestiones que se plantean:

\section{SITUACIÓN 1.}

Descripción 1 - Dos amigos acaban de estar tomando un café. Hacía algún tiempo que no se veían. Uno de ellos debe marcharse y es el que inicia la despedida. La persona que tiene que marcharse debe introducir de forma indirecta un motivo que va a servir como justificación para iniciar el ritual previo a la separación y despedida. Muchas veces puede ser una señal tan sutil como mirar el reloj.

Si su interlocutor no reconoce ese intento, se debe insinuar de nuevo, siendo cada vez más concreto. En el momento que el interlocutor reconoce que se van a despedir, se inicia el preludio a la separación: proponer un futuro encuentro, pero sin cerrarlo; respetar siempre que si uno de los participantes inicia un nuevo tema, su interlocutor debe continuarlo; enviar saludos a personas conocidas por ambos...

Descripción 2 - Dos amigos acaban de estar tomando un café. Hacía algún tiempo que no se veían. Uno de ellos debe marcharse y es el que inicia la despedida. Se lo comunica a su 
interlocutor y ambos inician la despedida promoviendo un posible encuentro para una próxima vez pero sin extender demasiado el intercambio.

1. Añade algún comentario, sugerencia u observación en relación a esta situación que justifique tu elección.

2. ¿Crees que podría variar tu elección dependiendo de la relación que tuvieses con la persona? Si tu respuesta es sí, explica por qué.

\section{SITUACIÓN 2.}

Descripción 1 - Un grupo de amigos acaba de cenar en un restaurante y llega el momento de pagar la cuenta. La persona que agarra la nota divide el total de la cuenta entre el número de personas que hay en la mesa, sin tener en cuenta qué ha consumido cada uno de ellos.

En alguna situación específica, si el grupo de amigos tiene una relación muy íntima, pueden producirse rituales de lucha por asumir el pago de la cuenta.

Descripción 2 - Un grupo de amigos acaban de cenar en un restaurante y llega el momento de pagar la cuenta. Cada persona paga su parte de la cuenta de acuerdo con lo que ha consumido.

1. Añade algún comentario, sugerencia u observación en relación a esta situación que justifique tu elección.

2. ¿Crees que podría variar tu elección dependiendo de la relación que tuvieses con las personas o el tipo de contexto (formal, reunión de estudiantes)? Si tu respuesta es sí, explica por qué.

\section{SITUACIÓN 3.}

Descripción 1 - Un compañero de trabajo le pide a otro compañero, al cual solo conoce del contexto laboral, si le puede ayudar a terminar un informe que debe presentar al día siguiente. Ante esta petición, su compañero se justifica diciéndole que no puede ayudarle porque tiene que hacer otra cosa. En su argumentación, se asegura de justificar debidamente y con motivos más que suficientes su negación. Se disculpa repetidas veces, al mismo tiempo que se asegura que su interlocutor ha aceptado la negación y que no la ha sentido como una agresión u ofensa.

Descripción 2 - Un compañero de trabajo le pide a otro compañero, al cual solo conoce del contexto laboral, si le puede ayudar a terminar un informe que debe presentar al día siguiente. Ante esta petición, su compañero le plantea otras opciones que le pueden ser útiles para terminar su informe. Si su interlocutor insiste, se va a ver obligado a decir siempre de forma indirecta que no va a poder, evitando en todo momento realizar una denegación clara y evidente.

1. Añade algún comentario, sugerencia u observación en relación a esta situación que justifique tu elección. 